\title{
Antifungal efficacy of itraconazole-loaded TPGS-b-(PCL-ran-PGA) nanoparticles
}

\author{
This article was published in the following Dove Press journal: \\ International Journal of Nanomedicine \\ 17 February 2015 \\ Number of times this article has been viewed
}

\author{
Lixin Qiu ${ }^{1,2, *}$ \\ Bicheng $\mathrm{Hu}^{1,4, *}$ \\ Hongbo Chen ${ }^{2,3}$ \\ Shanshan $\mathrm{Li}^{5}$ \\ Yuqian $\mathrm{Hu}^{2,3}$ \\ Yi Zheng ${ }^{2,3}$ \\ Xinxing $\mathrm{Wu}^{\prime}$
}

'Institute of Virology, School of Medicine, State Key Laboratory of Virology, Wuhan University, Wuhan, Hubei, People's Republic of China; ${ }^{2}$ The Shenzhen Key Lab of Gene and Antibody Therapy, Center for Biotech and Biomedicine, Division of Life and Health Sciences, Graduate School at Shenzhen, Tsinghua University, Shenzhen, Guangdong, People's Republic of China; ${ }^{3}$ School of Life Sciences, Tsinghua University, Beijing, People's Republic of China; ${ }^{4}$ The Clinical Laboratory, Wuhan No I Hospital, Wuhan, Hubei, People's Republic of China; ${ }^{5}$ Department of Plant Pathology and Microbiology, lowa State University, Ames, IA, USA

*These authors contributed equally to this work

Correspondence: Yi Zheng

Shenzhen Key Lab of Gene and Antibody

Therapy, Center for Biotech and

Biomedicine, Division of Life and Health

Sciences, Graduate School at Shenzhen,

L40I, Tsinghua Campus, Xili University

Town, Shenzhen 518055, Guangdong,

People's Republic of China

Tel +86755 26036068

Fax +8675526036736

Email zhengy@sz.tsinghua.edu.cn

\section{Xinxing Wu}

Institute of Virology, School of Medicine, State Key Laboratory of Virology, Wuhan University, Donghu Road II5, Wuchang

District, Wuhan 43007I, Hubei, People's

Republic of China

Tel +86 2768758898

Fax +86 2768758766

Email wuxinxing9755@163.com
Abstract: This research was conducted to formulate biodegradable itraconazole (ITZ)-loaded D-a-tocopheryl polyethylene glycol 1000 succinate-b-poly(e-caprolactone-ran-glycolide) (TPGSb-(PCL-ran-PGA); TPP) nanoparticles (NPs) (designed as ITZ-loaded TPP NPs) to improve antifungal efficacy. ITZ-loaded TPP NPs were prepared by a modified double-emulsion method, and their size distribution, morphology, zeta potential, drug encapsulation efficiency, drug-release profile, and antifungal effects were characterized. The cytotoxicity of ITZ-loaded-TPP NPs on HeLa cells and fibroblasts was measured using the 3-(4,5-dimethylthiazol-2-yl)-2,5- diphenyltetrazolium bromide (MTT) method. The in vivo antifungal activity of ITZ-loaded-TPP NPs was examined in mice by administrating $5 \times 10^{5}$ colony forming units of Candida albicans through the tail vein. The survival rate and survival time of the mice was observed. The fungal count and pathology of lung tissue was analyzed. The data showed that ITZ-loaded-TPP NPs have size of $265 \pm 5.8 \mathrm{~nm}$, zeta potential of $-31 \pm 0.5 \mathrm{mV}$, high encapsulation efficiency (95\%), and extended drug-release profile. ITZ-loaded-TPP NPs at a high concentration of $25 \mathrm{mg} / \mathrm{mL}$ had no cytotoxicity on HeLa cells and fibroblasts. Furthermore, ITZ-loaded-TPP NPs achieved a higher level of antifungal activity both in vitro and in vivo. The survival rate and duration was higher in mice treated by ITZ-loaded-TPP NPs than in the other groups $(P<0.05)$. In conclusion, ITZ-loaded-TPP NPs significantly improved ITZ bioavailability by increasing its aqueous dispersibility and extending the duration of drug release, thereby improving the antifungal efficacy of the ITZ agent.

Keywords: antifungal, Candida albicans, itraconazole (ITZ), nanoparticles (NPs), TPGS-b(PCL-ran-PGA) (TPP)

\section{Introduction}

Fungal diseases tend to occur most often as opportunistic infections in patients with a weakened immune system, such as patients with human immunodeficiency virus infection, diabetes mellitus, or leukemia, or in patients who received immunosuppressive therapy or chemotherapy. ${ }^{1}$ The symptoms of fungal infection vary with location within the body and the type of infection. Some fungal infections can be mild. However, some types of fungal infections, such as Candida spp., can result in serious complications or even death. ${ }^{2}$ Currently, many kinds of new drugs, such as polyenes (amphotericin B), triazoles (fluconazole, itraconazole, voriconazole, posaconazole) or echinocandins (caspofungin, micafungin, anidulafungin), have been specifically developed to inhibit fungal infection. ${ }^{3-5}$ Among these drugs, itraconazole (ITZ) is one of the most promising for the prophylaxis of opportunistic fungal infections in patients, due to its excellent ability to inhibit a wide range of superficial and deep fungal infections. ${ }^{6}$ ITZ can be used to treat fungal infections via oral or intravenous administration. ${ }^{7}$ However, ITZ has low bioavailability via oral administration owing to its very low aqueous solubility of approximately $1 \mathrm{ng} / \mathrm{mL}$ at $\mathrm{pH} 7.0 .^{8,9}$ The current 
commercial intravenous formulation of ITZ, formulated with a high concentration of hydroxypropyl-beta-cyclodextrin, has been found to have some side effects, including diarrhea. ${ }^{10-13}$ Therefore, less-toxic, solvent-free formulations of ITZ for effective antifungal therapy are urgently needed for clinical administration.

Recently, polymeric nanoparticles (NPs) have been widely investigated as drug carriers due to their ability to effectively deliver water-insoluble drugs, reduce the drug blood concentration fluctuations, and obtain persistent drug release. ${ }^{14}$ In the past decade, several biodegradable aliphatic polyesters, such as poly(e-caprolactone) (PCL) and polyglycolide (PGA) have been approved by the US Food and Drug Administration for use in the field for drug delivery. ${ }^{15,16}$ However, the high crystallinity of PCL results in poor soft tissue compatibility, lower biodegradability, and a slower degradation rate than PGA. PGA is also not a perfect material for a NP drug delivery system because of its high melting temperature of about $220^{\circ} \mathrm{C}$, high crystallinity of $45 \%-55 \%$, and insolubility in common solvent. ${ }^{16-18}$ These drawbacks have hampered the application of PCL and PGA in NP drug delivery systems.

To solve these problems, we have previously synthesized a novel diblock copolymer, D-a-tocopheryl polyethylene glycol 1000 succinate-b-poly(e-caprolactone-ran-glycolide) [TPGS-b-(PCL-ran-PGA)] using PCL, PGA, and D- $\alpha-$ tocopheryl polyethylene glycol 1000 succinate (TPGS) by ring-opening polymerization reaction. ${ }^{19} \mathrm{We}$ found that TPGS-b-(PCL-ran-PGA) (TPP)-based NPs has better biocompatibility, biodegradability, dispersibility, drug encapsulation efficiency, and drug-release profile than PGA and PCL NPs alone. ${ }^{19}$ In this study, we prepared the ITZ-loaded TPP NPs by a modified double-emulsion method and investigated the NP size, morphology, drug-release profile, and antifungal effects in vitro and in vivo to explore this novel antifungal agent.

\section{Materials and methods Materials}

TPGS, glycolide (1,4-Dioxane-2,5-dione), polyvinyl alcohol (80\% hydrolyzed), 3-(4,5-dimethylthiazol-2-yl)2,5-diphenyltetrazolium bromide (MTT), ITZ, poly(lacticco-glycolic acid) (PLGA) (50:50) and stannous octoate were purchased from Sigma-Aldrich (St Louis, MO, USA). $\varepsilon$-Caprolactone was from Acros Organics (Geel, Belgium). Acetonitrile and methanol were purchased from EM Science (ChromAR, HPLC grade; Mallinckrodt Baker, USA). Dulbecco's Modified Eagle's Medium (DMEM) and fetal bovine serum were from Invitrogen (Thermo Fisher
Scientific, Waltham, MA, USA). All other agents were of analytical grade or higher quality and were commercially available. High-purity water was prepared using a Milli-Q plus System (Millipore Corporation, Breford, USA). All experiments were performed in strict accordance with the Guide for the Care and Use of Laboratory Animals as adopted by the China National Institutes of Health, and the animal experiments were approved by the Administrative Committee on Animal Research in the Graduate School at Shenzhen, Tsinghua University (No TSUSZ20130012).

\section{Preparation of ITZ-loaded TPP NPs}

TPP polymers were synthesized according to a previously described method. ${ }^{19}$ Then ITZ-loaded TPP NPs were prepared by a modified double emulsion method. Briefly, $100 \mathrm{mg}$ TPP polymers and $6.4 \mathrm{mg}$ ITZ were fully dissolved in $10 \mathrm{~mL}$ dichloromethane (DCM). The formed solution was poured into $50 \mathrm{~mL}$ of $3 \%(\mathrm{w} / \mathrm{v})$ polyvinyl alcohol solution under vigorous stirring. The mixture was sonicated for 10 minutes with pulses of 10 seconds on and 10 seconds off at $600 \mathrm{~W}$ output to form oil/water emulsion. DCM was then allowed to evaporate overnight under continuous stirring at $500 \mathrm{rpm}$. The suspension was then dialyzed in a Regenerated Cellulose Dialysis Membrane (Spectra/Por 6, molecular weight cutoff $=1,000$; Spectrum, Houston, TX, USA) to remove the emulsifier and unencapsulated ITZ. The dialysis bag was put into ultrapure water under gentle stirring for 12 hours, with water changed every 4 hours. The resulting suspension was freeze-dried at $-50^{\circ} \mathrm{C}$ at a reduced pressure of $0.13 \mathrm{~Pa}$ for 48 hours. The resulting NPs were stored at $-80^{\circ} \mathrm{C}$ for further analysis. The preparation of ITZ-loaded PLGA NPs was similar to the methods described above. ${ }^{19}$ Empty TPP NPs were also prepared via the same procedure, with the exception that no ITZ was added to the DCM.

\section{NP characterization}

The surface morphology, size, zeta potential, drug encapsulation efficiency (EE) and drug release profile of nanoparticles are pivotal characterizations, which determine the functions.

\section{Surface morphology}

Samples were dropped onto double-sided sticky tape on the stub. The particles were then coated with a platinum layer using a JFC-1300 automatic fine platinum coater (JEOL, Tokyo, Japan) for 30 seconds in a vacuum. The surface morphologies of the NPs were observed by a 
field emission scanning electron microscope (JSM-6700F system; JEOL).

\section{Particle size and zeta potential}

Particle size and size distribution were measured by dynamic light scattering (Brookhaven Instruments Corporation, Holtsville, NY, USA). Zeta potential of the NPs was determined by using laser Doppler anemometry (Zetasizer Nano ZS90; Malvern Instruments LTD, Malvern, UK). Each measurement was performed in triplicate.

\section{Encapsulation efficiency}

Fifteen milligrams of ITZ was dissolved in $5 \mathrm{~mL}$ 95:5 (v/v) acetonitrile:water solution and diluted in 10 -fold series $(0$, $0.003,0.03,0.3,3 \mathrm{mg} / \mathrm{mL}$ ). Then the ITZ standard curve was prepared by measuring the light absorption value at $300 \mathrm{~nm}$ with a spectrophotometer (UV 1601; Shimadzu, Kyoto, Japan). The amount of ITZ encapsulated in the NPs was calculated by dissolving $5 \mathrm{mg}$ of the freeze-dried NPs in $5 \mathrm{~mL}$ of acetonitrile:water solution, and ITZ concentration was determined according to the standard curve. The ITZ encapsulation efficiency was defined as the ratio of the amount of ITZ encapsulated in the NPs to that theoretically added in the fabrication process.

\section{In vitro release of ITZ from NPs}

Thirty milligrams of ITZ-loaded NPs was suspended in $10 \mathrm{~mL}$ of phosphate-buffered saline (PBS) release buffer (containing $0.05 \% \mathrm{w} / \mathrm{v}$ Tween $80, \mathrm{pH} 7.4$ ).${ }^{19}$ The dispersion was then aliquoted into ten tubes and shaken at $37^{\circ} \mathrm{C}$ and $150 \mathrm{rpm}$. At appropriate intervals, the supernatant was collected by centrifuging the tube at $15,000 \mathrm{rpm}$ for 30 minutes. The ITZ concentration in the supernatant was determined as described in the encapsulation efficiency (EE) method.

\section{Cytotoxicity assay}

The cytotoxicity of ITZ-loaded TPP NPs was evaluated using the MTT assay. Briefly, HeLa cells or fibroblasts were seeded at a density of $5 \times 10^{3}$ cells/well in $100 \mu \mathrm{L}$ culture medium into a 96-well plate and incubated overnight. The cells were incubated with different concentrations of NPs for 48 hours and 72 hours. At indicated time points, the culture medium was replaced with $100 \mu \mathrm{L}$ MTT $(5 \mathrm{mg} / \mathrm{mL}$, in DMEM medium) and the plates were incubated at $37^{\circ} \mathrm{C}$ for another 4 hours; thereafter, MTT was removed and DMSO was added to completely dissolve the formazan crystals. Absorbance at $570 \mathrm{~nm}$ was measured with a microplate reader (Varioskan Flash; Thermo Fisher Scientific).

\section{In vitro antifungal effects}

The in vitro antifungal activity was tested using the paperplate technique. Briefly, $10 \mu \mathrm{L}$ of conidial suspension at $1 \times 10^{5}$ spores $/ \mathrm{mL}$ of Candida albicans (ATCC 10231) was spread over the glucose agar plate; $5 \mathrm{~mm}$ diameter filter paper was made with a sterile borer and was sterilized. Three pieces of sterile filter paper were placed on the solidified agar layer. Four different treatments $(10 \mu \mathrm{L})$ were dropped onto the papers: PBS, ITZ-loaded PLGA NP (equivalent to $0.3 \mathrm{mg} / \mathrm{mL}$ ITZ), ITZ-free TPP NP suspensions at $10 \mathrm{mg} / \mathrm{mL}$, and ITZ-loaded TPP NP suspensions at $10 \mathrm{mg} / \mathrm{mL}$ (equivalent to $0.3 \mathrm{mg} / \mathrm{mL}$ of ITZ), respectively. The plates were then incubated at $28^{\circ} \mathrm{C}$ for 7 days. The plates were photographed and the inhibitory zones were measured every day.

\section{In vivo antifungal effects}

\section{Survival test of mice after treatment}

$\mathrm{BALB} / \mathrm{c}$ mice were randomly divided into five groups with ten animals each group: group 1 (noninfected group with no treatment); group 2 (infected group with PBS treatment); group 3 (infected group with ITZ-loaded PLGA NPs treatment); group 4 (infected group with ITZ-free TPP NPs treatment); group 5 (infected group with ITZ-loaded TPP NPs treatment). Mice were challenged intravenously via the tail vein with $50 \mu \mathrm{L}$ of $1 \times 10^{7}$ colony forming units $(\mathrm{CFU}) / \mathrm{mL} C$. albicans, and then the mice were given corresponding administration with $100 \mu \mathrm{L}$ relevant solution $(100 \mu \mathrm{L}$ ITZ-loaded PLGA NP or ITZ-loaded TPP NP suspension is equivalent to $0.03 \mathrm{mg}$ ITZ) via tail vein 12 hours after challenge of $C$. albicans. On day 3 (48 hours after first administration), the mice were given corresponding administration once again, and the behaviors and lifetime of mice were observed every day. In this animal experiment, we begin to calculate the survival of mice after the first administration of drug. All surviving mice were euthanized on day 16 .

\section{Fungi count test and pathological examination}

On day 3, three mice of each group were euthanized by cervical dislocation 48 hours after the first administration. We aseptically removed their lungs for our experiments because the size of NPs is about $260 \mathrm{~nm}$, which is easily retained in lungs, and their lungs were severely damaged. The right lung was rinsed and homogenized in sterile PBS with a tissue grinder. Then, the suspension was diluted to 100 multiples and was cultured on a glucose agar plate. The CFUs were counted to determine the CFU/g of tissue (ie, colony forming units per gram of lung tissue). These colonies were further analyzed by yeast morphology assay and $18 \mathrm{~S}$ 
rDNA polymerase chain reaction analysis. In addition, the left lung tissue was embedded with paraffin and sectioned at $5 \mu \mathrm{m}$. Hematoxylin/eosin staining was performed to observe the pathological change.

\section{Statistical analysis}

Raw data were analyzed using the SPSS statistical software (version 16.0, IBM Corporation, Armonk, NY, USA). All data are expressed as means \pm standard deviation of three independent experiments. Differences between paired groups were analyzed by analysis of variance. $P<0.05$ was considered statistically significant.

\section{Results}

\section{Size, zeta potential, and drug encapsulation efficiency}

Size distribution, zeta potential, drug-loading content and encapsulation efficiency are summarized in Table 1. As shown in Table 1, TPP NPs have a diameter of about $265 \mathrm{~nm}$. Although there is a little difference between the NP size as detected by scanning electron microscopy and dynamic light scattering, the difference was not significant. Zeta potential is a marker of stability of NPs in suspension. The detection by laser Doppler anemometry showed that the zeta potential of TPP NPs was $-31 \mathrm{mV}$, which contributed to the dispersivity and stability of the NPs. The amount of ITZ encapsulated in the TPP and PLGA NPs was $10.04 \%$ and $9.7 \%$, respectively, and the drug encapsulation efficiency of both NPs was up to $95 \%$.

\section{Surface morphology}

Figure 1 shows the field emission scanning electron microscopy image of the ITZ-loaded TPP NPs. The NPs seemed to be around $260 \mathrm{~nm}$ in diameter with a smooth surface within the resolution level. This size is consistent with the result described above.

\section{In vitro drug release}

The drug-release profile of the ITZ-loaded NPs in the first 12 days is shown in Figure 2. The kinetics curve of ITZloaded NPs displays a biphase feature. The ITZ release from the TPP NPs was about $23.75 \%$ and $56 \%$ of the encapsulated drug in the first 3 days and after 12 days, respectively, suggesting that TPP NPs can serve as a drug reservoir to realize the long-acting antifungal effects of ITZ, similar to ITZ-loaded PLGA NPs. However, the ITZ release from TPP NPs was more rapid than that from PLGA NPs, although the difference was not significant $(P>0.05)$.

\section{Cytotoxicity of ITZ-loaded TPP NPs}

To determine whether ITZ-loaded TPP NPs are cytotoxic for cells, the in vitro cell viability of ITZ-loaded TPP NPs was evaluated by the MTT assay using HeLa cells and fibroblasts. As shown in Figure 3, the ITZ-loaded TPP NPs had no obvious cytotoxic activity at $0.25 \mathrm{mg} / \mathrm{mL}$ and $25 \mathrm{mg} / \mathrm{mL}$ concentrations after 24 hours and 48 hours incubation, suggesting that the ITZ-loaded TPP NPs are safe for use.

\section{The in vitro antifungal activity}

The antifungal activity of four different treatments (PBS control, ITZ-loaded PLGA NPs, ITZ-free TPP NPs, and ITZ-loaded TPP NPs) were tested by paper-plate technique, and the diameters of inhibition zones were measured. ITZfree TPP NPs had no visible inhibitory activity against fungal growth (Figure 4A) compared with the PBS control (Figure 4B). ITZ-loaded PLGA showed moderate antifungal effect, with inhibition-zone diameters of $9.43 \pm 0.25 \mathrm{~mm}$ on day $3,10.61 \pm 0.15 \mathrm{~mm}$ on day 5 , and $11.85 \pm 0.25 \mathrm{~mm}$ on day 7 , indicating that the diameters of the inhibition zone of agent application did increase as time progressed (Figure 4C). In particular, ITZ-loaded TPP NPs showed superior antifungal efficacy compared to ITZ-loaded PLGA NPs, with inhibition zone diameters of $11.43 \pm 0.25 \mathrm{~mm}$ on day $3,13.67 \pm 0.15 \mathrm{~mm}$ on day 5 , and $15.32 \pm 0.21 \mathrm{~mm}$ on day 7 (Table 2), which present clear inhibition zones beyond the areas of application (Figure 4D).

\section{The in vivo antifungal activity}

To evaluate the antifungal activity of ITZ-loaded TPP NPs in vivo, mice were challenged intravenously via the tail vein with $50 \mu \mathrm{L}$ of $1 \times 10^{7} \mathrm{CFU} / \mathrm{mL}$ C. albicans and then administered PBS, ITZ-loaded PLGA NPs, ITZ-free-TPP NPs,

Table I Characterization of NPs

\begin{tabular}{lllll}
\hline NP type & Size $(\mathbf{n m})$ & Zeta potential $(\mathbf{m V})$ & Drug loading $(\%)$ & EE\% \\
\hline TPP NPs & $260 \pm 4.7$ & $-29 \pm 0.4$ & & \\
ITZ-TPP NPs & $265 \pm 5.8$ & $-31 \pm 0.5$ & $10.04 \pm 0.3$ & $95 \pm 3.6$ \\
ITZ-PLGA NPs & $260 \pm 6.2$ & $-28 \pm 0.47$ & $9.7 \pm 0.29$ & $95 \pm 2.8$ \\
\hline
\end{tabular}

Abbreviations: EE, encapsulation efficiency; ITZ, itraconazole; NP, nanoparticle; PLGA, poly(lactic-co-glycolic acid); TPP, TPGS-b-(PCL-ran-PGA). 


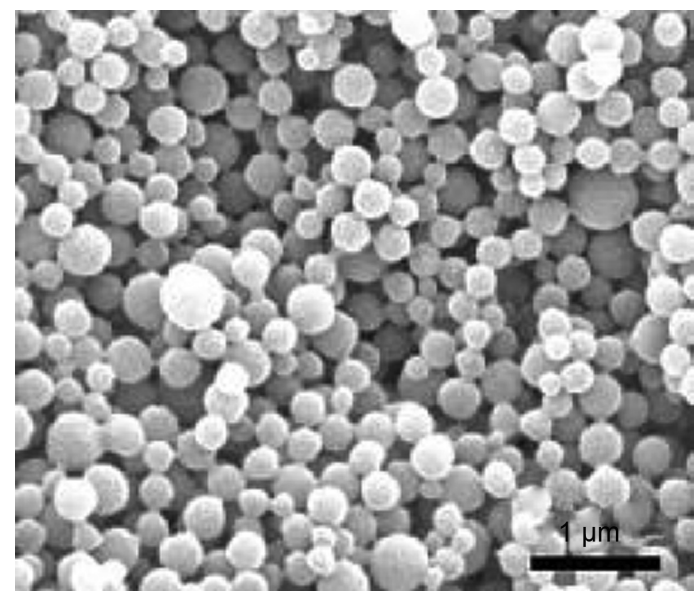

Figure I Field emission scanning electron photomicrograph of ITZ-loaded TPP NPs. Note: The results showed that ITZ-loaded TPP NPs are round, and the size is around $260 \mathrm{~nm}$.

Abbreviations: ITZ, itraconazole; NP, nanoparticle; TPP, TPGS-b-(PCL-ran-PGA).

and ITZ-loaded TPP NPs. On day 3, 48 hours after the first administration, the lung tissue of three mice in each group was homogenized for the fungi count test and the $\mathrm{CFU} / \mathrm{g}$ of tissue was calculated. As shown in Table 3, the group with ITZ-loaded PLGA NPs treatment had a significantly lower value of $1.6 \times 10^{3} \mathrm{CFU} / \mathrm{g}$ of tissue compared with those of the PBS control group and ITZ-free TPP NPs group $(P<0.01)$. In particular, treatment of ITZ-loaded TPP NPs showed

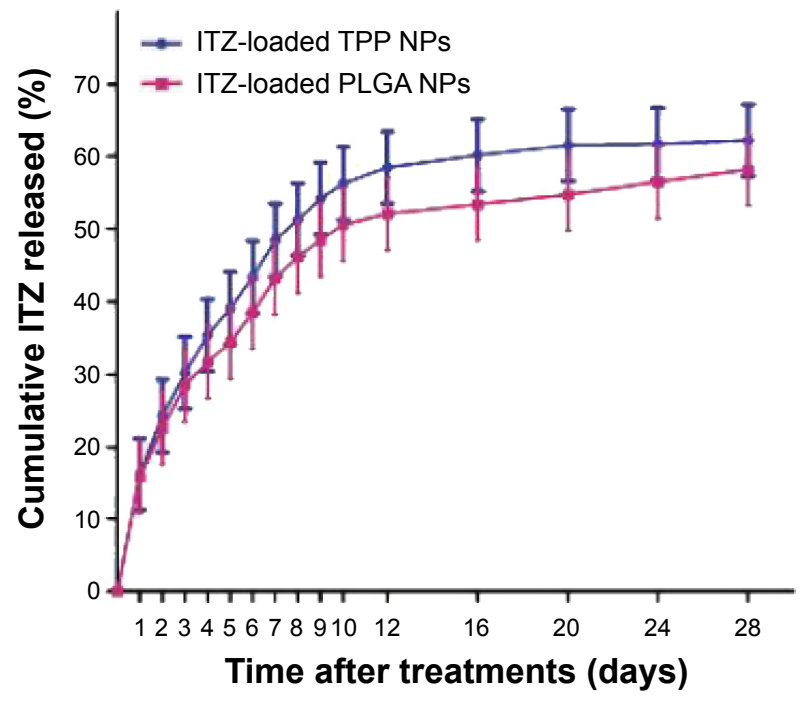

Figure 2 In vitro release profiles of ITZ from loaded TPP NPs and PLGA NPs. Notes: There was no significant difference $(P>0.05)$ between ITZ-loaded TPP NPs and ITZ-loaded PLGA NPs. However, the release efficacy of ITZ-loaded TPP NPs was higher than that of ITZ-loaded PLGA NPs at all indicated times. The release curve has biphase kinetics: at the initial phase, the release is burst-like; then the release is relatively stable. The release of ITZ-loaded TPP NPs and ITZ-loaded PLGA NPs was up to $50 \%$ at day 8 and day 10 , respectively. The experiment was performed in $\mathrm{PBS}$ at $37^{\circ} \mathrm{C}$.

Abbreviations: ITZ, itraconazole; NP, nanoparticle; PBS, phosphate-buffered saline; PLGA, poly(lactic-co-glycolic acid); TPP, TPGS-b-(PCL-ran-PGA).

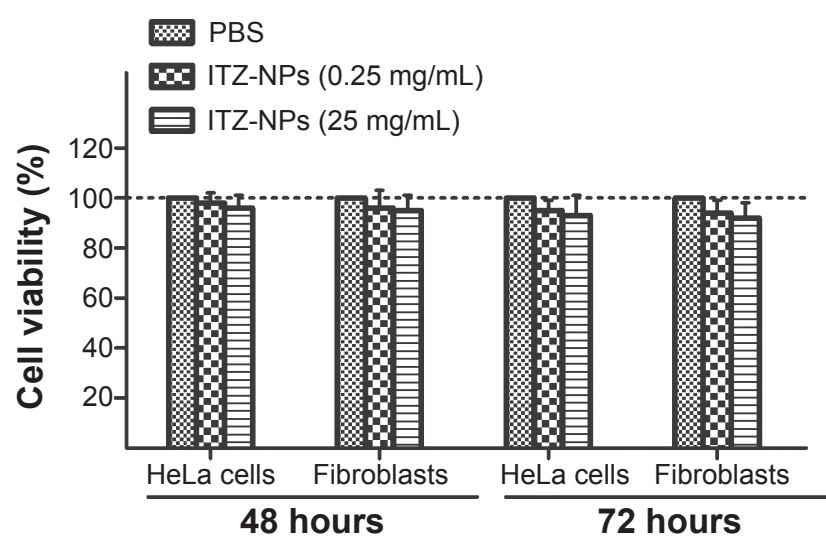

Figure 3 Viability of HeLa cells and fibroblasts treated with ITZ-loaded TPP NPs. Notes: The results showed that ITZ-loaded TPP NPs had no cytotoxic activity on HeLa cells and fibroblasts at dosages of $0.25 \mathrm{mg} / \mathrm{mL}$ and $25 \mathrm{mg} / \mathrm{mL}$ at 48 hours and 72 hours.

Abbreviations: ITZ, itraconazole; NP, nanoparticle; PBS, phosphate-buffered saline; TPP, TPGS-b-(PCL-ran-PGA).

the best inhibitory efficacy against $C$. albicans growth in lung of mice, with a value of $1.2 \times 10^{2} \mathrm{CFU} / \mathrm{g}$, much less than $2.4 \times 10^{6} \mathrm{CFU} / \mathrm{g}$ in mice treated by ITZ-free TPP NPs. C. albicans could be detected in lung of mice treated by ITZ-loaded PLGA NPs, but it was rarely detected in mice treated with ITZ-loaded TPP NPs. The mice treated with ITZfree NPs tended to gather and be torpid, and to have slower responses and lower activity levels. Their lung tissues displayed blood dots, and their pulmonary alveoli contained red cells and a large amount of inflammatory cells (Figures 5B and D). Meanwhile, mice in the ITZ-loaded PLGA NPs group only exhibited a pathological behavior. The lung epidermis cells were impaired, pulmonary alveoli contained red cells, and less inflammatory-cell infiltration compared with the PBS and ITZ-free NPs groups (Figure 5C). It is encouraging that mice treated with ITZ-loaded TPP NPs displayed normal behavior and that their lung tissues had normal pathological features (Figures 5A and E). The survival rate and duration of mice has been observed and the data indicated that ITZloaded TPP NPs had the best protective effects against fungal infection in mice (Figure 6).

\section{Discussion}

Polymer-based NPs have been widely investigated as a drug-delivery system, especially for hydrophobic drugs. ${ }^{20}$ However, the biodegradable polymers NPs that have been approved by the US Food and Drug Adminstration, such as PCL and PLGA, have poor compatibility and a too-slow degradation rate in vivo owing to their compaction structure. ${ }^{16-18}$ Several newly synthesized polymers have been developed to improve NP drug-delivery systems. In our previous study, 

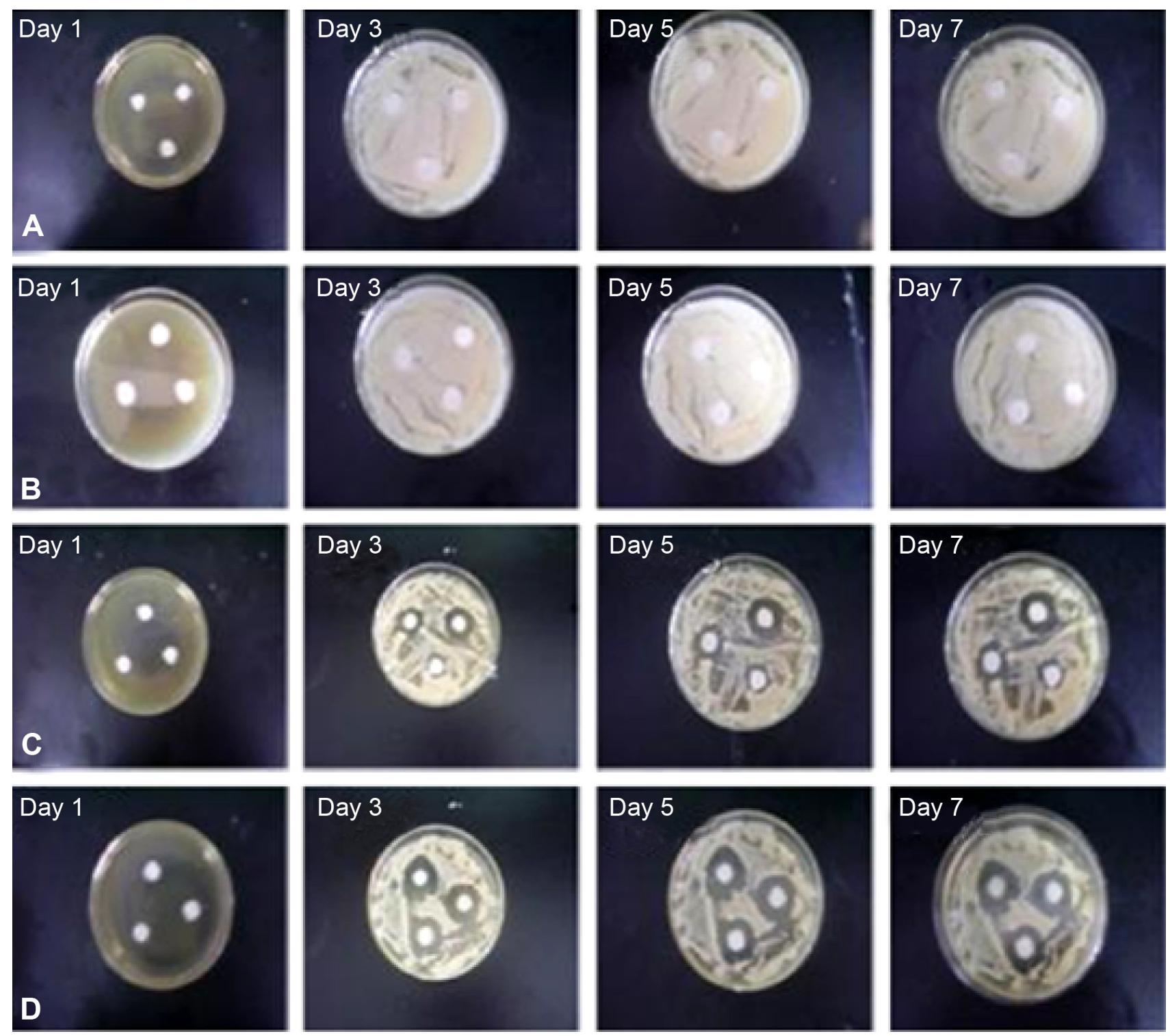

Figure 4 Antifungal activity of four different treatments (PBS control, ITZ-loaded PLGA NPs, ITZ-free TPP NPs, and ITZ-loaded TPP NPs).

Notes: Paper-plate growth inhibition of Candida albicans treated with (A) ITZ-free TPP NPs; (B) PBS; (C) ITZ-loaded PLGA NPs; and (D) ITZ-loaded TPP NPs. The results showed that ITZ-free TPP NPs and PBS had no inhibitory effect on C. albicans, whereas ITZ-loaded TPP NPs and ITZ-loaded PLGA NPs could inhibit the growth of $C$. albicans. In addition, the inhibitory zone of ITZ-loaded TPP NPs and ITZ-loaded PLGA NPs showed that the inhibitory effect of ITZ-loaded TPP NPs on C. albicans was superior than that of ITZ-loaded PLGA NPs, which probably results from the drug release difference of ITZ-loaded TPP NPs and ITZ-loaded PLGA NPs.

Abbreviations: ITZ, itraconazole; NP, nanoparticle; PBS, phosphate-buffered saline; PLGA, poly(lactic-co-glycolic acid); TPP, TPGS-b-(PCL-ran-PGA).

Table 2 Diameters of the inhibition zones of drug application on day 7

\begin{tabular}{ll}
\hline Treatments & $\begin{array}{l}\text { Diameters } \\
(\mathbf{m m})\end{array}$ \\
\hline PBS control & 0.0 \\
ITZ-loaded PLGA NPs & $11.85 \pm 0.25$ \\
ITZ-free TPP NPs & 0.0 \\
ITZ-loaded TPP NPs & $15.32 \pm 0.21 *$ \\
\hline
\end{tabular}

Note: $* P<0.05$ (versus ITZ-loaded PLGA NPs).

Abbreviations: ITZ, itraconazole; NP, nanoparticle; PBS, phosphate-buffered saline; PLGA, poly(lactic-co-glycolic acid); TPP, TPGS-b-(PCL-ran-PGA).
Table 3 The CFU/g lung tissue of mice treated by different agents $(n=3)$

\begin{tabular}{ll}
\hline Treatments & CFU/g \\
\hline PBS control & $2.8 \times 10^{6}$ \\
ITZ-loaded PLGA NPs & $1.6 \times 10^{3 *}$ \\
ITZ-free TPP NPs & $2.4 \times 10^{6}$ \\
ITZ-loaded TPP NPs & $1.2 \times 10^{2 \dagger}$ \\
\hline
\end{tabular}

Notes: $* P<0.01$ ITZ-loaded PLGA NPs versus PBS control and empty NPs. ${ }^{\dagger P}<0.01$ ITZ-loaded TPP NPs versus the other controls.

Abbreviations: CFU, colony forming unit; ITZ, itraconazole; NP, nanoparticle; PBS, phosphate-buffered saline; PLGA, poly(lactic-co-glycolic acid); TPP, TPGS-b-(PCLran-PGA) 


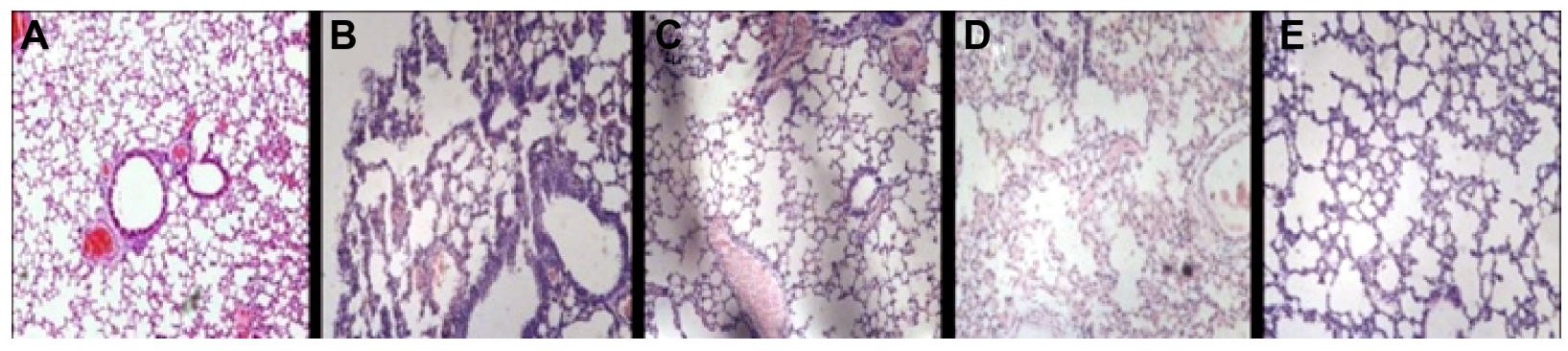

Figure 5 The pathological change in lung tissue for mice treated with different administrations.

Notes: (A) Normal mouse, untreated. (B) Infected group with PBS treatment. (C) Infected group with ITZ-loaded PLGA treatment. (D) Infected group with ITZ-free TPP NPs treatment. (E) Infected group with ITZ-loaded TPP NPs treatment.

Abbreviations: ITZ, itraconazole; NP, nanoparticle; PBS, phosphate-buffered saline; PLGA, poly(lactic-co-glycolic acid); TPP, TPGS-b-(PCL-ran-PGA).

a novel polymer, TPP, was synthesized and confirmed to have better advantage in biocompatibility, biodegradability, dispersibility, docetaxel-encapsulation efficiency, and docetaxel-release profile. ${ }^{19}$

In this work, the ITZ-loaded NPs were prepared using the TPP material for antifungal infection. Our results showed that ITZ-to-polymer ratios at 5:1 can obtain low particle size of about $265 \mathrm{~nm}$ with high encapsulation efficiency of $95 \%$. Previous research reported that TPGS addition in the manufacture of NPs can promote the EE of water-insoluble

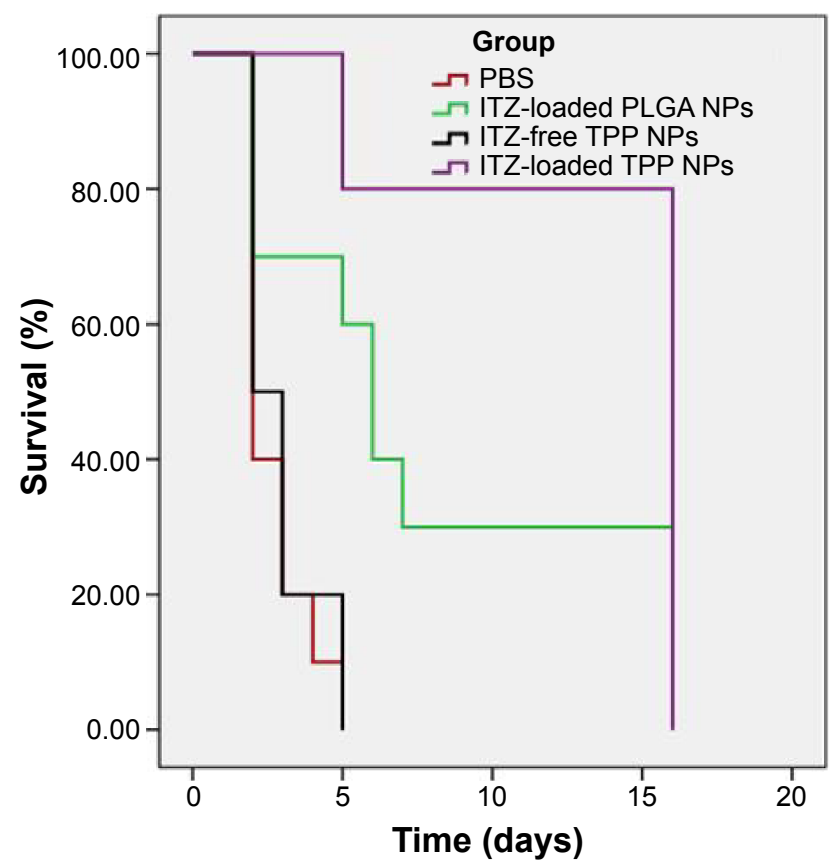

Figure 6 The survival test curves for mice under different treatments $(n=10 /$ group).

Notes: All PBS-treated mice and mice treated with ITZ-free TPP NPs died within 6 days. In contrast, $80 \%$ of the mice survived after administration of ITZ-loaded TPP NPs and $30 \%$ survived after treatment with ITZ-loaded PLGA NPs; the survival time was more than 15 days. In this animal experiment, all surviving mice were euthanized on day 16.

Abbreviations: ITZ, itraconazole; NP, nanoparticle; PBS, phosphate-buffered saline; PLGA, poly(lactic-co-glycolic acid); TPP, TPGS-b-(PCL-ran-PGA). drugs and decrease the size of NPs; thus, the low particle size and high EE of ITZ-loaded TPP NPs may be caused by the self-emulsifying function of TPGS. ${ }^{21}$ In addition, TPGS has been widely used as an emulsifier in the manufacture of NPs, offering additional steric stabilization effect while avoiding aggregation of NPs in the colloidal system, which can promote the stability of the particles. ${ }^{22}$ Zeta potential is an important physicochemical parameter that influences stability of the NPs. Similar positive or negative zeta potential values can prevent aggregation of the nanoparticles and ensure good redispersion. ${ }^{23}$ The zeta potential of ITZ-loaded TPP NPs is $-31 \mathrm{mV}$, indicating that it is desirable result.

The antifungal activity of the developed NPs formulation was further examined in vitro by the paper-plate test. ITZ-loaded TPP NPs demonstrated an enhanced inhibitory effect compared with ITZ-loaded-PLGA NPs (Figure 4 and Table 2). The enhanced antifungal activity may be attributed to the following reasons. First, TPP NPs were expected to improve the dispersing of ITZ in water. As previously mentioned, ITZ is a water-insoluble drug, which results in its low bioavailability. Since TPP NPs are hydrophilic and small in size, this facilitates their contact with the fungus on the plate surface and leads to faster drug internalization. Secondly, TPP NPs constitute a slow-release drug delivery system and can prevent drugs from degradation, similar to PLGA-based vectors (Figure 2). While ITZ is consumed or degraded, the fungus near the point of drug application continues to grow. The sustained release of ITZ from TPP NPs can provide a constant inhibition on fungal growth, resulting in larger inhibition zones as observed over the time of the study. This will help reduce frequencies of drug administration, therefore reducing the side effects.

The in vivo study also demonstrated that ITZ-loaded TPP NPs have a better therapeutic effect for fungal infection than ITZ-loaded PLGA NPs (Figures 5 and 6). The mice treated with ITZ-loaded TPP NPs had better behaviors and 
lower death rates than the mice in the ITZ-loaded PLGA NPs group. In this work, we did not compare the antifungal efficacy of ITZ-loaded TPP NPs to ITZ alone. Instead, we compared the antifungal efficacy of ITZ-loaded TPP NPs to ITZ-loaded PLGA NPs. It has been reported by Patel et $\mathrm{al}^{24}$ that the antifungal efficacy of ITZ-loaded PLGA NPs is superior to ITZ alone. Based on these facts, we think that the application of ITZ-loaded TPP NPs is better than the traditional ITZ treatment. Lung is the most common target organ of fungal infection in some cases, such as AIDS patients. ${ }^{25,26}$ Because previous studies have reported that NPs with a size of 100-1,000 $\mathrm{nm}$ are able to be retained in the lung, ${ }^{27,28}$ it is highly likely that ITZ-loaded TPP NPs could accumulate in the lung tissue. In addition, the release of TPGS from NPs may play some role. Detailed mechanism needs further investigation.

\section{Conclusion}

We developed the ITZ-loaded TPP NPs with appropriate particle size, high EE, and an extended drug-release profile. The ITZ-loaded TPP NPs have been proven to be an effective means for enhancing the antifungal effects of ITZ. ITZ-loaded TPP NPs provide a better choice for patients with fungal diseases when traditional antifungal agents are not available or do not work.

\section{Acknowledgments}

This research was supported by the National Natural Science Foundation of China (No 81072123) and the Clinical Medical Research Project of Wuhan Health and Family Planning Commission (WX13A01). The animal experiments were approved by the Administrative Committee on Animal Research in Graduate School at Shenzhen, Tsinghua University (No TSUSZ20130012).

\section{Disclosure}

The authors report no conflicts of interest in this work.

\section{References}

1. Pagano L, Akova M, Dimopoulos G, Herbrecht R, Drgona L, Blijlevens N. Risk assessment and prognostic factors for mould-related diseases in immunocompromised patients. $J$ Antimicrob Chemother. 2011;66 Suppl 1:i5-i14.

2. Enoch DA, Ludlam HA, Brown NM. Invasive fungal infections: a review of epidemiology and management options. J Med Microbiol. 2006; 55(Pt 7):809-818.

3. Jung SH, Lim DH, Jung SH, et al. Amphotericin B-entrapping lipid nanoparticles and their in vitro and in vivo characteristics. Eur J Pharm Sci. 2009;37(3-4):313-320.

4. Odds FC, Brown AJ, Gow NA. Antifungal agents: mechanisms of action. Trends Microbiol. 2003;11(6):272-279.
5. Sabatelli F, Patel R, Mann PA, et al. In vitro activities of posaconazole, fluconazole, itraconazole, voriconazole, and amphotericin B against a large collection of clinically important molds and yeasts. Antimicrob Agents Chemother. 2006;50(6):2009-2015.

6. Panda NK. Itraconazole - a potent antifungal drug. Indian J Otolaryngol Head Neck Surg. 1997;49(3):293-294.

7. Willems L, van der Geest R, de Beule K. Itraconazole oral solution and intravenous formulations: a review of pharmacokinetics and pharmacodynamics. J Clin Pharm Ther. 2001;26(3):159-169.

8. Peeters J, Neeskens P, Tollenaere JP, Van Remoortere P, Brewster ME. Characterization of the interaction of 2-hydroxypropyl-b-cyclodextrin with itraconazole at $\mathrm{pH} \mathrm{2,} \mathrm{4,} \mathrm{and} \mathrm{7.} \mathrm{J} \mathrm{Pharm} \mathrm{Sci.} \mathrm{2002;91(6):}$ 1414-1422.

9. De Beule K. Itraconazole: pharmacology, clinical experience and future development. Int J Antimicrob Agents. 1996;6(3):175-181.

10. Akkar A, Müller RH. Intravenous itraconazole emulsions produced by SolEmuls technology. Eur J Pharm Biopharm. 2003;56(1):29-36.

11. Chen W, Gu B, Wang H, Pan J, Lu W, Hou H. Development and evaluation of novel itraconazole-loaded intravenous nanoparticles. Int J Pharm. 2008;362(1-2):133-140.

12. Yi Y, Yoon HJ, Kim BO, et al. A mixed polymeric micellar formulation of itraconazole: characteristics, toxicity and pharmacokinetics. $J$ Control Release. 2007;117(1):59-67.

13. Gould S, Scott RC. 2-Hydroxypropyl-beta-cyclodextrin (HP-beta-CD): a toxicology review. Food Chem Toxicol. 2005;43(10):1451-1459.

14. Panyam J, Labhasetwar V. Biodegradable nanoparticles for drug and gene delivery to cells and tissue. Adv Drug Deliv Rev. 2003;55(3): 329-347.

15. Mundargi RC, Srirangarajan S, Agnihotri SA, et al. Development and evaluation of novel biodegradable microspheres based on poly(D,Llactide-co-glycolide) and poly(epsilon-caprolactone) for controlled delivery of doxycycline in the treatment of human periodontal pocket: in vitro and in vivo studies. J Control Release. 2007;119(1): $59-68$.

16. Vert M. Degradable and bioresorbable polymers in surgery and in pharmacology: beliefs and facts. J Mater Sci Mater Med. 2009;20(2): 437-446.

17. Andriano KP, Daniels AU, Smutz WP, Wyatt RW. Preliminary biocompatibility screening of several biodegradable phosphate fiber reinforced polymers. J Appl Biomater. 1993;4(1):1-12.

18. Sun H, Mei L, Song C, Cui X, Wang P. The in vivo degradation, absorption and excretion of PCL-based implant. Biomaterials. 2006;27(9): $1735-1740$.

19. Huang $\mathrm{L}$, Chen $\mathrm{H}$, Zheng $\mathrm{Y}$, et al. Nanoformulation of $\mathrm{D}-\alpha$-tocopheryl polyethylene glycol 1000 succinate-b-poly( $\varepsilon$-caprolactone-ranglycolide) diblock copolymer for breast cancer therapy. Integr Biol (Camb). 2011;3(10):993-1002.

20. Matsumura Y, Kataoka K. Preclinical and clinical studies of anticancer agent-incorporating polymer micelles. Cancer Sci. 2009;100(4): $572-579$.

21. Ruan G, Feng SS, Li QT. Effects of material hydrophobicity on physical properties of polymeric microspheres formed by double emulsion process. J Control Release. 2002;84(3):151-160.

22. Mu L, Feng SS. Vitamin E TPGS used as emulsifier in the solvent evaporation/extraction technique for fabrication of polymeric nanospheres for controlled release of paclitaxel (Taxol). J Control Release. 2002;80(1-3):129-144.

23. Mainardes RM, Evangelista RC. PLGA nanoparticles containing praziquantel: effect of formulation variables on size distribution. Int $J$ Pharm. 2005;290(1-2):137-144

24. Patel NR, Damann K, Leonardi C, Sabliov CM. Itraconazole-loaded poly(lactic-co-glycolic) acid nanoparticles for improved antifungal activity. Nanomedicine (Lond). 2010;5(7):1037-1050.

25. Pacheco P, Ventura AS, Branco T, Gonçalves L, Carvalho C. Clinical experience in invasive fungal infections. Clin Drug Investig. 2013;33 Suppl 1:S23-S26. 
26. Gazzoni FF, Severo LC, Marchiori E, et al. Fungal diseases mimicking primary lung cancer: radiologic-pathologic correlation. Mycoses. 2014;57(4):197-208.

27. Borak B, Biernat P, Prescha A, Baszczuk A, Pluta J. In vivo study on the biodistribution of silica particles in the bodies of rats. Adv Clin Exp Med. 2012;21(1):13-18.
28. Wanakule P, Liu GW, Fleury AT, Roy K. Nano-inside-micro: diseaseresponsive microgels with encapsulated nanoparticles for intracellular drug delivery to the deep lung. J Control Release. 2012;162(2): 429-437.

\section{Publish your work in this journal}

The International Journal of Nanomedicine is an international, peerreviewed journal focusing on the application of nanotechnology in diagnostics, therapeutics, and drug delivery systems throughout the biomedical field. This journal is indexed on PubMed Central, MedLine, CAS, SciSearch $®$, Current Contents $® /$ Clinical Medicine,
Journal Citation Reports/Science Edition, EMBase, Scopus and the Elsevier Bibliographic databases. The manuscript management system is completely online and includes a very quick and fair peer-review system, which is all easy to use. Visit http://www.dovepress.com/ testimonials.php to read real quotes from published authors.

Submit your manuscript here: http://www.dovepress.com/international-journal-of-nanomedicine-journal 